\title{
Spontaneous intermuscular hematoma in a patient receiving ticagrelor and aspirin: A case report
}

\author{
BYUNG WOO YOON ${ }^{1,2}$ and JI YEON HONG ${ }^{3}$ \\ ${ }^{1}$ Department of Internal Medicine, Division of Hemato-Oncology, Seoul Paik Hospital, Seoul 04551; \\ ${ }^{2}$ Inje University, College of Medicine, Busan 47392; \\ ${ }^{3}$ Department of Internal Medicine, Division of Cardiology, Nowon Eulji Medical Center, Eulji University, \\ Seoul 01830, Republic of Korea
}

Received October 15, 2020; Accepted December 14, 2020

DOI: $10.3892 /$ br. 2020.1404

\begin{abstract}
Dual antiplatelet therapy is a cornerstone treatment following percutaneous coronary intervention (PCI) for acute coronary syndrome (ACS). Ticagrelor, a direct-acting potent P2Y12 inhibitor, is superior to clopidogrel in the acute setting of ACS: due to its faster onset and potent antiplatelet inhibition. Thus, ticagrelor is becoming widely recommended as the as the initial treatment following PCI in current guidelines. However, due to its potency, the risk of bleeding is higher. Herein, a report of an unusual case of spontaneous intermuscular hematoma is described. Furthermore, single-nucleotide polymorphisms that may be related to bleeding in ticagrelor using population-based genome-wide association studies were also identified and are discussed. As there is a concern that patients of Asian origin may have a higher risk of bleeding, physicians must be made aware of this risk when prescribing ticagrelor to individuals of Asian decent.
\end{abstract}

\section{Introduction}

Dual antiplatelet therapy (DAPT) is a cornerstone treatment following percutaneous coronary intervention (PCI) for acute coronary syndrome (1). Ticagrelor, a direct-acting potent P2Y12 inhibitor, is superior to clopidogrel, due its faster onset and more potent antiplatelet inhibitory effect, and is becoming increasingly used as the initial medication administered following PCI in current guidelines (2-4). Ticagrelor is an active metabolite when absorbed, whereas clopidogrel requires two steps to become an active metabolite. However, due to the stronger potency and rapid onset of effects of ticagrelor, the

Correspondence to: Professor Ji Yeon Hong, Department of Internal Medicine, Division of Cardiology, Nowon Eulji Medical Center, Eulji University, 68 Hangeulbiseok-Ro, Nowon-Gu, Seoul 01830, Republic of Korea

E-mail: gladyshong@gmail.com

Key words: ticagrelor, spontaneous intermuscular hematoma, genome-wide association studies risk of bleeding is higher: Although the rates of life threatening bleeding are similar, non-procedure-related bleeding (Gastrointestinal bleeding) is higher in patients administered ticagrelor $(3,4)$. In the present report, a case of spontaneous intermuscular hematoma, which may be due to administration of ticagrelor is described. Furthermore, single-nucleotide polymorphisms (SNPs) that may be related to bleeding in ticagrelor using population-based genome-wide association studies (GWAS) were also identified and are discussed.

\section{Case report}

A 69-year-old Korean man with a 5-year history of exertional chest pain, 10-year history of hypertension (on medication), 10 -year history of dyslipidemia, a heavy current smoker (20 pack-years), and a heavy drinker (one bottle of soju every other day) visited our outpatient clinic at Nowon Eulji Medical Center, Eulji University. His chest pain had worsened during the previous 7 days, and he had diaphoresis with pain radiating to the left arm during his sleep. His vital signs were: Blood pressure of $150 / 80 \mathrm{mmHg}$, heart rate of $78 \mathrm{bpm}$, respiratory rate of 20 breaths $/ \mathrm{min}$, and a temperature of $36.8^{\circ} \mathrm{C}$. There were no abnormalities during physical examination. Laboratory tests showed no abnormalities, except for elevated triglycerides (410 $\mathrm{mg} / \mathrm{dl})$, and there was no elevation in creatinine kinase myocardial band and troponin-I levels. The prothrombin time (PT) was $10.6 \mathrm{sec}$ (reference value, 10-13 sec), PT international normalized ratio (INR) was 0.91 (reference value, 0.85-1.15) and activated partial thromboplastin time (aPTT) was $28.6 \mathrm{sec}$ (reference value, 25-36 sec). His electrocardiogram was normal, and a 2D-echocardiography test was performed to show a normal left ventricle (LV) ejection fraction of $67 \%$, normal LV cavity size, and normal LV contractility without regional wall motion abnormality. Due to his aggravating chest pain history with combined symptoms during the resting period, he was diagnosed with unstable angina. Upon coronary angiography, $80 \%$ stenosis was found in the middle to distal left anterior descending (LAD) artery, with a normal right coronary artery. PCI was initiated, and a $2.5 \times 24 \mathrm{~mm}$ Biomatrix stent was implanted in the middle to distal LAD. After PCI, the patient was administered $100 \mathrm{mg}$ aspirin once daily, $90 \mathrm{mg}$ ticagrelor twice daily, $1.25 \mathrm{mg}$ bisoprolol once daily, $10 \mathrm{mg}$ lercanidipine 

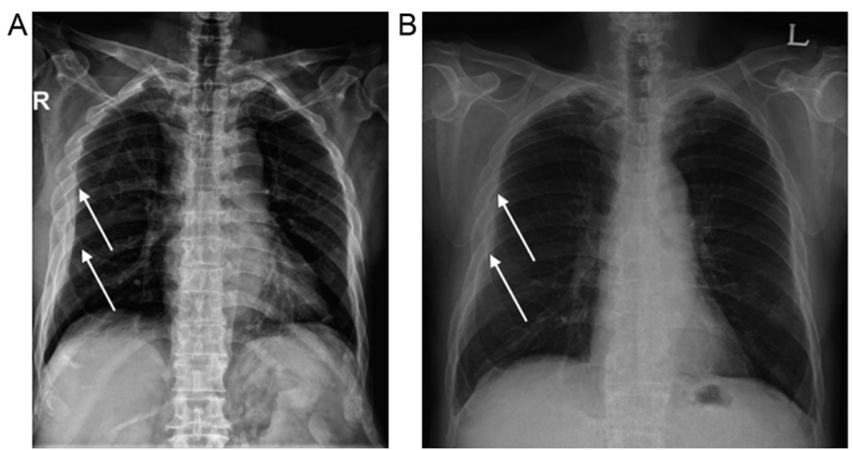

Figure 1. Chest radiograph images. (A) Chest radiograph when the patient visited the emergency room, presenting with right-chest pain and bruising shown in the right back and axilla. (B) Chest radiograph after primary coronary intervention. Arrows mark the area of the corresponding intermuscular hematoma.
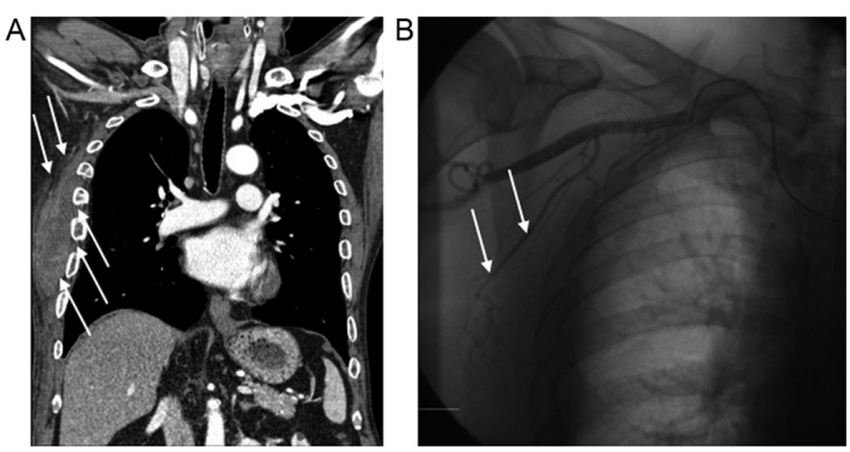

Figure 2. Chest computed tomography and angiography images. (A) Corona image of a chest computed tomography, showing a hematoma in the right chest area. (B) Thoracic angiography image on the right lateral thoracic artery, showing no arterial bleeding. Arrows mark the area of the corresponding intermuscular hematoma.

once daily and $20 \mathrm{mg}$ rosuvastatin once daily. The patient was discharged on the third day without complications.

However, a month after discharge, the patient visited the emergency department (ED) with abrupt pain and bruising in the right chest wall and axilla area, which caused him to wake up from his sleep. He denied trauma, particularly in the right chest area. His electrocardiogram and cardiac enzymes were normal, and laboratory tests showed no abnormalities except a sudden drop in hemoglobin levels from 14.6 to $10.0 \mathrm{mg} / \mathrm{dl}$, while his platelet level was $248,000 / \mathrm{mm}^{3}$, PT level $11.8 \mathrm{sec}$, PT INR 1.01 and aPTT $28.6 \mathrm{sec}$. His chest radiograph (CXR) showed subcutaneous haziness in the right chest area with swelling (Fig. 1A). An immediate chest computed tomography scan showed an intermuscular hematoma in the right subcutaneous lesion and fracture at the 4th, 5th, 6th and 7th ribs (Fig. 2B), although the patient strongly denied a recent history of trauma. An immediate thoracic angiography was performed on the right lateral thoracic artery and multiple right intercostal arteries, showing no arterial or venous bleeding (Fig. 2B). On comparing the CXR after PCI (Fig. 1B) and the CXR after visiting the ED (Fig. 1A), a bone deformity was initially seen in the post-PCI CXR (Fig. 1A). An extensive history of the patient was obtained, where he admitted that he had right rib fractures 4 months prior to PCI intervention and 5 months prior to his ED visit. Therefore, intermuscular hematoma at the right chest wall was concluded to be caused by spontaneous bleeding from ticagrelor and aspirin, as there is no evidence of hematoma from healing bone fractures. After discontinuation of aspirin and ticagrelor for 1 week, the patient was restarted on DAPT: $100 \mathrm{mg}$ of aspirin once a day and $75 \mathrm{mg}$ of clopidogrel once a day for 4 months. On the follow-up outpatient clinic visit, there were no symptoms of ischemia nor bleeding events.

Written informed consent was obtained from the patient for publication of this case report and any accompanying images.

\section{Discussion}

In the present case, the patient was not categorized as having a high risk of bleeding. According to the hypertension, abnormal renal/liver function, stroke, bleeding history or predisposition, labile INR, elderly, drugs/alcohol (HAS-BLED) scoring system (5). The HAS-BLED score of the patient was 2 due to being aged $>65$-years and his current use of medication, which is under the high-risk value of $\leq 3$. Thus, when the patient presented to the ED with right back pain and bruising, it was initially assumed that the large intermuscular hematoma was due to recent trauma. However, scrutiny of the post-PCI CXR and the CXR during his ED visit showed that fractures were initially present, after which the patient admitted that he had rib fractures 4 months to PCI; however, the possibility of spontaneous hematoma is very low from healing rib fractures (6). Furthermore, thoracic angiography of the right lateral thoracic artery and multiple right intercostal arteries showed that there was no evidence of recent trauma leading to arterial or venous rupture. Therefore, by ruling out other causes of bleeding, it was concluded that the ticagrelor was the cause of spontaneous bleeding.

According to the Platelet Inhibition and Patient Outcomes (PLATO) trial, ticagrelor exhibits superior efficacy compared with clopidogrel in reducing cardiovascular events and mortality at the expense of increased risk of non-fatal bleeding (3). However, ethnicity was not considered in this trial, and Asians are known to have a higher risk of being susceptible to bleeding complications when administered antithrombotics or fibrinolytics (7-10). Kang et al (11) performed a retrospective analysis of the PLATO trial and showed that there was no significant differences in the risk of major, fatal or minor bleeding between Asian and non-Asian patients when they were on ticagrelor or clopidogrel. Nevertheless, there is a doubt as to whether the bleeding events of ticagrelor are lower than that of clopidogrel, particularly in the Asian population. Furthermore, there are no large-randomized trials that have been performed in the Asian population to evaluate adverse events, to the best of our knowledge.

In particular, when searching for other reported cases of intermuscular hematoma, only one case was documented in China. Feng et al (12) reported a similar case in which the patient had a spontaneous hematoma under his right scapular site in the setting of ticagrelor and aspirin after PCI without a history of trauma.

The limitation of the present report is the lack of bleeding time (BT) and platelet function tests (PFT) when the patient visited the ED. BT and PFT are not routine laboratory tests performed in Korea as these tests are difficult to perform in acute settings, such as in the ED or before PCI. BT requires skilled staff from the department of laboratory medicine, and they can be hard to 


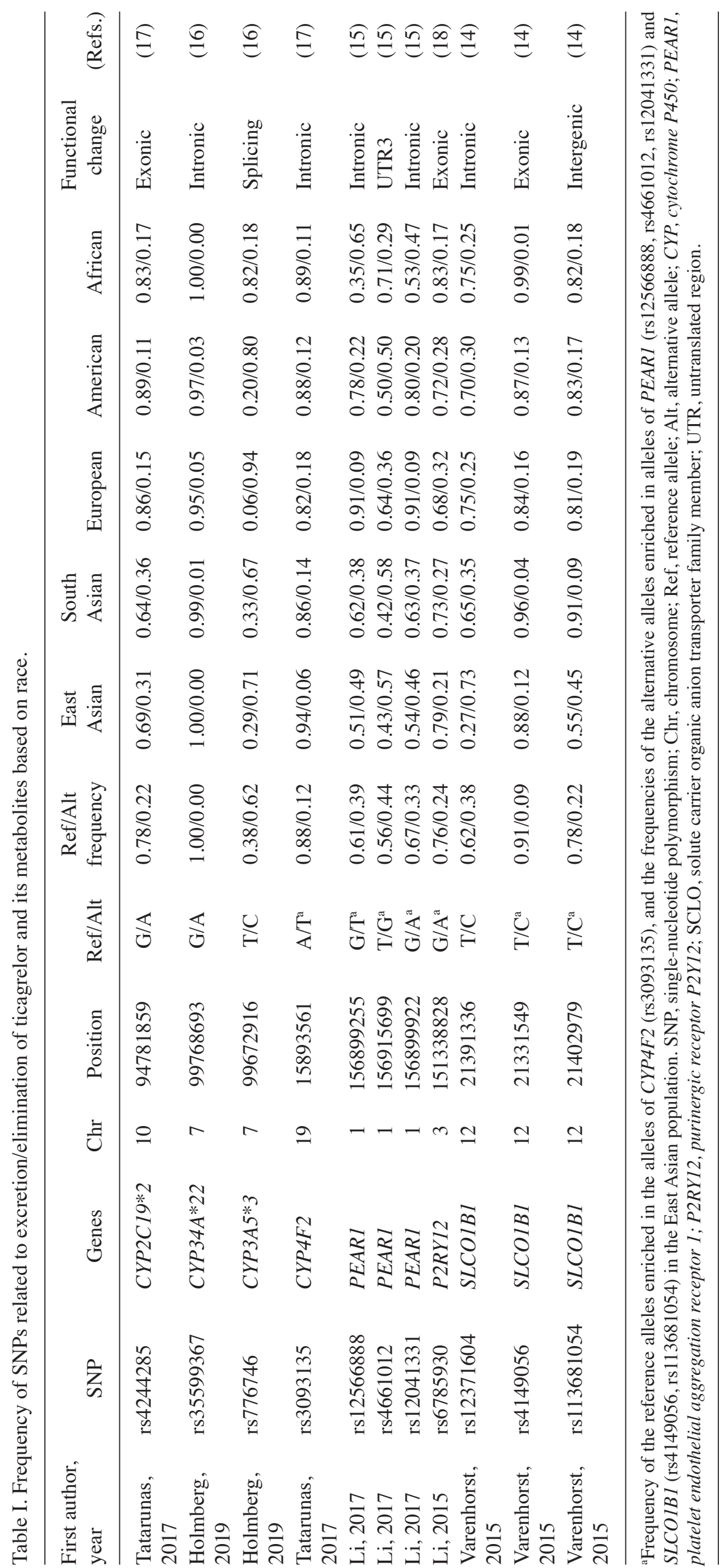


request on-call. PFT is reported the next day, providing limited information in an acute setting of bleeding. Furthermore, as these tests are not reimbursed by the Health and Welfare Ministry of Korea, patients need to pay at their own expense, which is hard to explain and request in an acute setting. However, comparing BT and PFT before and after ticagrelor may have provided additional information regarding the tendency of bleeding in our patient, as PT and aPTT time before and after ticagrelor and aspirin were both in the normal range. Considering that genetics may have some role in bleeding, we searched the GWAS catalog (13) and found that Varenhorst et al (14) performed GWAS on the PLATO trial to determine whether genetic variations may cause variations in ticagrelor plasma levels and clinical outcomes. Their study showed that ticagrelor pharmacokinetics were associated with SLCO1B1, UGT2B7 and CYP3A4; however, these associations did not translate into any detectable effect on efficacy or safety correlating to ticagrelor treatment (14). Li et al (15) showed that SCLO1B1 and CYP3A4/5 polymorphisms did not affect the pharmacokinetics and pharmacodynamics of ticagrelor treatment in healthy Chinese male subjects. Holmberg et al (16), also showed that although $C Y P 3 A 4 * 22$ (rs35599367 G>A) impairs elimination, it has no effect on the bioactivation of clopidogrel, and population-based genomics show that there is almost no variation in the frequency of $C Y P 3 A 4 * 22$ based on populations of diverse ancestry, and thus cannot explain the increased risk of bleeding in Asians.

Tatarunas et al (17) demonstrated that the CYP4F2 rs3093135 TT allele has a higher antiplatelet effect of ticagrelor and more frequent nonprocedural bleeding during ticagrelor therapy, as compared with AA and AT variant carriers. Additionally, $C Y P 2 C 19 * 1$ (rs4244285 G allele) has a higher antiplatelet effect than $C Y P 2 C 19 * 2$ (rs4244285 A allele). However, when reviewing the frequency of SNPs related to bleeding in the Korean/East Asian population, the mentioned SNPs could not explain the higher risk of bleeding in Asians.

Li et al (18) documented two SNP locations in the PEARI genes that were related to increased antiplatelet activity: rs12041311 (AA homozygotes) and rs4661012 (GG homozygotes). In terms of frequency, the AA frequency for rs12041311 is $16 \%$ in Koreans and $21 \%$ in East Asians, whereas it was $10 \%$ for the global population. In addition, the GG frequency of rs4661012 is 25\% in Koreans and 32\% in East Asians, whereas globally it is $20 \%$. Hence, a detailed pharmacogenetics study of rs12041311 and rs4661012 is required to improve our understanding of the pharmacokinetics and pharmacodynamics of these gene variants to evaluate the proper dose and adverse events of ticagrelor in the East Asian population. The SNPs evaluated from the GWAS are shown in Table I.

In conclusion, the present case report showed that ticagrelor, a potent antiplatelet inhibitor, may cause spontaneous intermuscular bleeding. As there is a concern that Asians may have a higher risk of bleeding, physicians must be aware of this risk when prescribing ticagrelor in the Asian population. However, further research is required to validate the association of the potential SNPs, particularly the PEARI gene and its relation to risk of bleeding risks amongst individuals of different races.

\section{Acknowledgements}

Not applicable.

\section{Funding}

No funding was received.

\section{Availability of data and materials}

All data generated or analyzed during this study are included in this published article.

\section{Authors' contributions}

BWY contributed to the diagnosis, data curation and writing of the manuscript. JYH contributed to the conceptualization of the report and reviewed the manuscript. BWY and JYH confirm the authenticity of all the raw data. All authors have read and approved the final manuscript.

\section{Ethics approval and consent to participate}

Not applicable.

\section{Patient consent for publication}

Written informed consent was obtained from the patient for publication of this case report and any accompanying images.

\section{Competing interests}

The authors declare no competing interests.

\section{References}

1. Kurz DJ and Eberli FR: Medical therapy of coronary artery disease after percutaneous intervention. Curr Opin Pharmacol 13: 287-293, 2013.

2. Amsterdam EA, Wenger NK, Brindis RG, Casey DE Jr, Ganiats TG, Holmes DR Jr, Jaffe AS, Jneid H, Kelly RF, Kontos MC, et al: 2014 AHA/ACC Guideline for the Management of Patients with Non-ST-Elevation Acute Coronary Syndromes: A report of the American College of Cardiology/American Heart Association Task Force on Practice Guidelines. J Am Coll Cardiol 64: e139-e228, 2014. Erratum in: J Am Coll Cardiol 64: 2713-2714, 2014

3. Wallentin L, Becker RC, Budaj A, Cannon CP, Emanuelsson H, Held C, Horrow J, Husted S, James S, Katus H, et al; PLATO Investigators: Ticagrelor versus clopidogrel in patients with acute coronary syndromes. N Engl J Med 361: 1045-1057, 2009.

4. Husted S and van Giezen JJ: Ticagrelor: The first reversibly binding oral $\mathrm{P} 2 \mathrm{Y}_{12}$ receptor antagonist. Cardiovasc Ther 27: 259-274, 2009.

5. Pisters R, Lane DA, Nieuwlaat R, de Vos CB, Crijns HJ and Lip GY: A novel user-friendly score (HAS-BLED) to assess 1-year risk of major bleeding in patients with atrial fibrillation: The Euro Heart Survey. Chest 138: 1093-1100, 2010.

6. Sato N, Sekiguchi H, Hirose Y and Yoshida S: Delayed chest wall hematoma caused by progressive displacement of rib fractures after blunt trauma. Trauma Case Rep 4: 1-4, 2016.

7. Ross AM, Gao R, Coyne KS, Chen J, Yao K, Yang Y, Qin X, Qiao S and Yao M; TUCC Investigators: A randomized trial confirming the efficacy of reduced dose recombinant tissue plasminogen activator in a Chinese myocardial infarction population and demonstrating superiority to usual dose urokinase: The TUCC trial. Am Heart J 142: 244-247, 2001.

8. Bhatt DL, Paré G, Eikelboom JW, Simonsen KL, Emison ES, Fox KA, Steg PG, Montalescot G, Bhakta N, Hacke W, et al; CHARISMA Investigators: The relationship between $C Y P 2 C 19$ polymorphisms and ischaemic and bleeding outcomes in stable outpatients: The CHARISMA genetics study. Eur Heart J 33: 2143-2150, 2012 
9. Wang TY, Chen AY, Roe MT, Alexander KP, Newby LK, Smith SC Jr, Bangalore S, Gibler WB, Ohman EM and Peterson ED: Comparison of baseline characteristics, treatment patterns, and in-hospital outcomes of Asian versus non-Asian white Americans with non-ST-segment elevation acute coronary syndromes from the CRUSADE quality improvement initiative. Am J Cardiol 100: 391-396, 2007.

10. Dang MT, Hambleton J and Kayser SR: The influence of ethnicity on warfarin dosage requirement. Ann Pharmacother 39: 1008-1012, 2005.

11. Kang HJ, Clare RM, Gao R, Held C, Himmelmann A, James SK, Lim ST, Santoso A, Yu CM, Wallentin L, et al; PLATO Investigators: Ticagrelor versus clopidogrel in Asian patients with acute coronary syndrome: A retrospective analysis from the Platelet Inhibition and Patient Outcomes (PLATO) Trial. Am Heart J 169: 899-905.e1, 2015.

12. Feng C, Wang L and Wang L: Spontaneous hematoma in the setting of dual anti-platelet therapy with ticagrelor: A case report. Oncol Lett 12: 144-146, 2016.

13. Buniello A, MacArthur JAL, Cerezo M, Harris LW, Hayhurst J Malangone C, McMahon A, Morales J, Mountjoy E, Sollis E, et al: The NHGRI-EBI GWAS Catalog of published genome-wide association studies, targeted arrays and summary statistics 2019 . Nucleic Acids Res 47 (D1): D1005-D1012, 2019.

14. Varenhorst C, Eriksson N, Johansson $\AA$, Barratt BJ, Hagström E, Åkerblom A, Syvänen AC, Becker RC, James SK, Katus HA, et al; PLATO Investigators: Effect of genetic variations on ticagrelor plasma levels and clinical outcomes. Eur Heart J 36: 1901-1912, 2015.
15. Li M, Hu Y, Li H, Wen Z, Hu X, Zhang D, Zhang Y, Xiao J, Tang J and Chen X: No effect of SLCO1B1 and CYP3A4/5 polymorphisms on the pharmacokinetics and pharmacodynamics of ticagrelor in healthy chinese male subjects. Biol Pharm Bull 40: 88-96, 2017.

16. Holmberg MT, Tornio A, Paile-Hyvärinen M, Tarkiainen EK, Neuvonen M, Neuvonen PJ, Backman JT and Niemi M: CYP $3 A 4^{*} 22$ impairs the elimination of ticagrelor, but has no significant effect on the bioactivation of clopidogrel or prasugrel. Clin Pharmacol Ther 105: 448-457, 2019.

17. Tatarunas V, Kupstyte N, Zaliunas R, Giedraitiene A and Lesauskaite V: The impact of clinical and genetic factors on ticagrelor and clopidogrel antiplatelet therapy. Pharmacogenomics 18: 969-979, 2017.

18. Li MP, Tang J, Wen ZP, Zhang YJ,Zhang W, Zhou HH, Zhang ZL and Chen XP: Influence of $P 2 Y 12$ polymorphisms on platelet activity but not ex-vivo antiplatelet effect of ticagrelor in healthy Chinese male subjects. Blood Coagul Fibrinolysis 26: 874-881, 2015.

This work is licensed under a Creative Commons Attribution-NonCommercial-NoDerivatives 4.0 International (CC BY-NC-ND 4.0) License. 